\title{
Delincuencia y violencia en entornos residenciales de Santiago de $\mathrm{Chile}^{1}$
}

\author{
Ximena Tocornal ${ }^{2}$, Ricardo Tapia ${ }^{3}$ y Yuri Carvajal ${ }^{4}$
}

\begin{abstract}
RESUMEN
Las situaciones de delincuencia y violencia que se manifiestan en Santiago de Chile, ocurren en ciertos territorios con determinadas características urbanas y sociales. De acuerdo a estudios realizados en ciudades del hemisferio norte, entre las características sociales más relevantes a la hora de explicar la concentración territorial de delitos, y en especial de los delitos violentos, destacan aquellas como la cohesión social, el control social informal, el respeto a normas u otras similares que refieren a la calidad de las relaciones prosociales entre vecinos, más allá de las tradicionales variables de tipo estructural como son la concentración de desventajas socioeconómicas. En este trabajo se presentan y discuten mediciones empíricas de dichas variables, teniendo como unidad de análisis territorial, una muestra representativa de los entornos residenciales de Santiago de Chile.
\end{abstract}

Palabras clave: Seguridad ciudadana, barrios, delincuencia.

\begin{abstract}
Episodes of crime and violence in Santiago, Chile take place in given territories with certain urban and social characteristics. In accordance with studies performed in cities in the northern hemisphere, some of the most relevant social characteristics for explaining the territorial concentration of crime, and especially violent crimes, are social cohesion, informal social control, respect for the law, amongst others. These variables are related to the pro-social quality of relationships among neighbors, and are separate from traditional structural variables, such as the concentration of the socioeconomically disadvantaged. In this work we present and discuss empirical measures of these variables, measured in a representative sample of the residents of Santiago, Chile.
\end{abstract}

Key words: Citizen security; neighborhoods; crime.

\footnotetext{
1 Proyecto Anillos de Investigación en Ciencias Sociales SOC-09 "Crimen y Violencia Urbana: Aportes de la Ecología del Delito al diseño de Políticas Públicas". Artículo recibido el 30 de mayo de 2012, aceptado el 7 de mayo de 2013 y corregido el 9 de septiembre de 2013.
}

\footnotetext{
2 Facultad de Psicología, Universidad Diego Portales (Chile). E-mail: ximena.tocornal@udp.cl

3 Instituto de la Vivienda, Universidad de Chile (Chile). E-mail: rictapia@uchilefau.cl

4 Escuela de Salud Pública, Universidad de Chile (Chile). E-mail: ycarvajal@med.uchile.cl
} 
¿Qué tan seguros son los entornos residenciales en la ciudad de Santiago de Chile? ¿Son estos seguros o inseguros debido al diseño de los espacios públicos y privados o a las personas que en ellos habitan, o bien a ciertas características de las comunidades que allí se conforman? En otras palabras, ¿qué relevancia tendrán los aspectos físicos y simbólicos del lugar, así como las interrelaciones entre ambas dimensiones, en la conformación de "un barrio seguro"? Y aún más importante, ¿será posible sostener que cambiando las dinámicas de las comunidades sea viable influir en los índices delictuales tomando como unidad de análisis el espacio físico y simbólico del barrio residencial?

Tradicionalmente, delincuencia y violencia han sido estudiados empíricamente en su relación con variables sociales y económicas de tipo estructurales que remiten fenómenos de exclusión social, marginalidad y, finalmente, a pobreza e inequidad (por ejemplo, ingreso familiar, acceso al mercado laboral, nivel educacional, entre otras). Luego, se ha sostenido que la concentración de desventajas socioeconómicas es una dimensión significativa a la hora de explicar las manifestaciones delictuales y/o violentas (Fajnzylber et al., 1998; Buvinić et al., 1999). Varios de estos estudios se han hecho teniendo como unidad de observación, países (Gaviria \& Pagés, 2002; Moser \& Mcllwaine, 2004) y, más recientemente, ciudades (Moncada, 2013; Arias, 2006; World Bank, 2010), pero son muy escasas -y aún más en el caso de la realidad latinoamericana- las investigaciones donde la unidad de análisis es un territorio más pequeño y abarcable según la experiencia cotidiana, es decir, un espacio físico y simbólico relativamente equivalente a lo que para una persona sería su barrio.

No deja de ser llamativo que "del conjunto de barrios que comparten similares condiciones socioeconómicas y demográficas (nivel de ingreso, de escolaridad y similares), solo en algunos de ellos se instalan prácticas violentas específicas que se reflejan en altas tasas delictuales. Ello evidencia que (...) habría otros factores intervinientes propios de la comunidad, que probablemente las estrategias de intervención (de políticas públicas) no han logrado captar e integrar en sus diseños e implementación" (Olavarría et al., 2008: 31).
Así, las siguientes preguntas se vuelven centrales: ¿Por qué en algunos barrios se mantienen altos niveles de criminalidad y violencia? y ¿Por qué en estos territorios las políticas públicas son poco efectivas y resulta extremadamente difícil y costoso revertir la situación que allí se vive?

En este contexto, resulta pertinente reducir la unidad de análisis a territorios más pequeños que la ciudad para describir, explicar y elaborar propuestas para prevenir la delincuencia y la violencia, toda vez que, además, el sentimiento de inseguridad o la comisión de un delito violento están en estrecha relación con el espacio o lugar específico donde ocurren o se experimentan dichas situaciones (Sampson, 2013). Al respecto, este trabajo espera contribuir con información descriptiva en la identificación de condiciones del entorno residencial asociadas a altos niveles de compromiso delictual (contra la propiedad y las personas) y a otras formas de violencia e incivilidades que amenazan una convivencia respetuosa entre vecinos.

Luego de una breve revisión de la aproximación conceptual de este estudio, así como de la exposición de la metodología de investigación, los resultados descriptivos son presentados por entornos residenciales, en tanto unidad de análisis territorial, organizado en 6 conjuntos de variables:

1. aquellas relativas a la victimización, el número de delitos reportados, tanto aquellos ocurridos en el entorno residencial como fuera de estos, diferenciados por delitos violentos contra las personas y delitos contra la propiedad (vivienda y vehículos u objetos en su interior);

2. aquellas relativas a la percepción de seguridad, de violencia y desorden social en los entornos residenciales, así como evaluaciones de riesgo de los vecinos con respecto a la posibilidad de ser víctima de algún delito de asaltos, lesiones, amenazas, robos contra la vivienda y vehículos y objetos en su interior;

3. variables relacionadas con la concentración de desventajas económicas y habitacionales, tales como nivel de ingresos de los hogares, nivel de hacinamiento y allegamiento y proximidad con respecto a conjuntos de viviendas sociales; 
4. variables residenciales tales como años de residencia, satisfacción residencial y elección del barrio;

5. variables que miden la presencia de instituciones del Estado en el territorio (salud, educación, policías, municipios, áreas recreativas) y la satisfacción de los vecinos con respecto a los servicios prestados por ellas; $y$

6. variables que miden características de las relaciones sociales entre vecinos (confianza, cohesión, control social informal, interacción reciproca, entre otras).

De esta forma, se tratan a nivel descriptivo las principales asociaciones entre variables que dicen relación con las características de las relaciones sociales entre vecinos, los niveles y tipos de victimización, la concentración de algunas desventajas sociales (incluida la cercanía/lejanía con los conjuntos de viviendas sociales) y la presencia y satisfacción de los vecinos con respecto a las instituciones del Estado.

\section{Aproximaciones conceptuales a la violencia urbana desde América Latina}

La literatura especializada ha reconocido ampliamente el carácter multicausal de la violencia y la delincuencia, en términos de fenómenos complejos y multifacéticos (Buvinić et al., 1999; Concha-Eastman y Guerrero, 1999; Carrión, 2003; Roché, 2004). No hay una sino muchas variedades o manifestaciones de violencia. Por ello, las dificultades de definirla. No ocurre lo mismo con la noción de delito, puesto que ella está estipulada en las leyes de cada nación, siendo relativamente más fácil tipificar una determinada situación como delito (incluido el delito violento o crimen) que como violencia, siguiendo las interpretaciones de los códigos penales. No obstante, resulta poco recomendable proponer estrategias de prevención centradas, únicamente, en definiciones legales del delito. Mientras hay delitos que no implican el uso de violencia, hay violencia que no está tipificada como delito o crimen en las legislaciones.

Además, lo que para unos puede ser considerado violento, para otros no lo es. La vio- lencia tendría, entonces, un claro componente subjetivo y relativo al contexto en el que se le reconoce como tal, poniendo de manifiesto la relevancia del lugar en y desde el que se despliega y significa la conducta violenta. En este sentido, las conductas violentas se aprenden, y el primer lugar de aprendizaje puede ser el hogar, luego, el entorno residencial más inmediato, la escuela y el espacio público del barrio (la plaza, el parque, la esquina, el pasaje).

En América Latina, las manifestaciones de violencia suelen concentrarse en los sectores de las ciudades más pobladas, en cuyos contextos la suma de desventajas sociales, la segregación espacial y social y la débil presencia del Estado, permiten la generación de ambientes de desorganización social que se traducen en más violencia y criminalidad. La noción de desorganización social ha sido sugerida por la tradición criminológica de la Escuela de Chicago, y especialmente la vertiente ecológica, como hipótesis explicativa de por qué sectores residenciales equivalentes en cuanto a concentración de desventajas socioeconómicas no evidencian niveles de delincuencia y violencia equiparables.

La desorganización social sería un estado característico de las relaciones entre vecinos, producto de la falta de cohesión social y de la incapacidad de ejercer control social informal ante las conductas desviadas que erosionan la confianza y el respeto para con el otro. En palabras de Sampson (1995:198), la desorganización social corresponde a "la incapacidad de una estructura comunitaria de sostener los valores comunes de sus residentes y de mantener efectivos mecanismos de control" de modo tal que "la organización y la desorganización están indisolublemente relacionadas con el sistema de redes que facilitan o inhiben el control social".

Una dimensión muy relevante en este modelo es el nivel de cohesión que experimentan las personas en relación con su hábitat residencial, que incluye el nivel de identificación positiva con su lugar de residencia y el nivel de confianza entre los vecinos. Otra dimensión relevante corresponde al nivel de control social informal sobre conductas desviadas que pueden ejercer los vecinos entre 
sí, especialmente de los adultos con respecto a niños y jóvenes en los espacios públicos. Asimismo, la integración de la dimensión de cohesión y de control social informal, en tanto ambas suelen asociarse muy fuertemente, ha sido trabajada como eficacia colectiva. En otras palabras, comunidades locales con altos niveles de eficacia colectiva son aquellas con mayores desarrollos de mecanismos protectores ante la desorganización social.

Los criminólogos que suscriben la teoría de la desorganización social han acumulado durante décadas un volumen importante de evidencia sobre la relación de determinados contextos urbanos con la emergencia de conductas de riesgo y antisociales. No obstante, advierten que no es posible ni conveniente idealizar la noción de barrio o comunidad como si esta correspondiera a una red de relaciones de amistad incondicional y parentesco fuertemente establecidas. Por el contrario, las redes sociales fomentarían las condiciones bajo las cuales la eficacia colectiva puede surgir, pero ello no es suficiente para el ejercicio del control. Para Sampson y sus colaboradores (Sampson et al., 2002), reconocer esto, implica comprender que en el mundo moderno la "comunidad" no es necesaria para la satisfacción de necesidades personales o privadas, ni siquiera para las necesidades de sustento, las cuales irremediablemente están dispersas en el espacio. Más bien, la "comunidad" local permanece como relevante en tanto es un lugar para la realización de bienes públicos o comunes, tales como la seguridad pública, la limpieza del medio ambiente y la educación de los niños. Así cobran mayor importancia las medidas de confianza entre vecinos, entendida como aquella condición que permite que el comportamiento de los vecinos sea predecible y predictible, facilitando los intercambios recíprocos de ayuda mutua, así como las acciones para vigilancia colectiva sobre los entornos del barrio, situaciones que bien pueden redundar sobre mayores niveles de seguridad y menor número de delitos en tales áreas.

No obstante, la solidez de la hipótesis de la desorganización social, las investigaciones empíricas han estado centradas en determinadas ciudades norteamericanas o europeas. Ejemplo de esto son los trabajos de Robert Sampson ya citados, así como los de Shaw \&
McKay (1942), Cohen \& Felson (1979), Bursik (1988), y Mazerolle et al. (2010). Las diferencias con los contextos latinoamericanos son importantes y se expresan, por ejemplo, en la composición demográfica, en los menores niveles de desarrollo económico que implican una fuerte presencia de la economía informal y en la menor capacidad estatal para ejecutar planes y programas que reduzcan el delito, entre otros factores. En efecto, algunos investigadores latinoamericanos sostienen que la cohesión social no se expresa necesariamente en menores niveles delictivos (Manzano, 2009) ni tiene una alta correlación con la confianza en los vecinos (Villareal \& Da Silva, 2006) como se desprende de los estudios hechos en ciudades norteamericanas o europeas. En la misma línea, siguiendo a Núñez et al. (2012), en un estudio multinivel en Santiago de Chile, el control social informal y el temor al delito presentan una asociación positiva, de tal manera que cuando hay mayor temor al delito en un barrio, también se registra una percepción de que los vecinos ejercen mayor control social informal; el punto es que, al parecer, ese mecanismo no estaría siendo eficiente para disminuir el temor.

A nivel de ciudades, otros estudios latinoamericanos muestran que se registran importantes niveles de correlación entre barrios con desventajas sociales y la incidencia criminal violenta en estos sectores (Londoño, 1996; Portes y Hoffman, 2003; Arriagada y Godoy, 2000; Araya y Sierra, 2002; Escobar, 2012), tendiendo a demostrar que no es la pobreza por sí misma la que explica los niveles de criminalidad, pues interviene un conjunto de otros factores mediadores, cuyo punto de partida, necesario pero no suficiente, es la pobreza que luego deriva en la incapacidad de las familias y de la comunidad para ejercer un control social eficaz.

Un fenómeno significativo en la explicación de cómo se instalan y perpetúan los niveles de desorganización social en un determinado entorno residencial dice relación con las dinámicas de segregación social y espacial, por una parte, y la aceptación de una cultura de la ilegalidad, por otra. Katzman et al. (2003) analizan el caso de la ciudad de Montevideo. Los barrios populares registran altas concentraciones de precariedades, incluyendo niveles de segregación que se 
expresan en barrios socialmente homogéneos cuyo vecindario no puede ofrecerles un mejor acceso a información y contactos útiles. Las malas condiciones del vecindario estigmatizan a la población y dificultan adicionalmente su ya difícil acceso al mercado laboral. Además, las actividades que pueden generar empleo se han alejado de estos sectores reduciendo de esta manera las oportunidades. El contexto de marginalidad genera subculturas que legitiman nuevas formas de acceder a los bienes de consumo; finalmente, el contexto priva a los menores de líderes o modelos positivos en su etapa de desarrollo.

\section{Muestra e instrumentos}

Los datos que se discuten en este trabajo fueron obtenidos, en su mayor parte, de la aplicación de una encuesta a 5.860 hogares, agrupados en 242 entornos residenciales representativos de las comunas urbanas de la ciudad de Santiago. La aplicación de la encuesta se realizó entre agosto y octubre de 2010.

Además, en cada uno de los entornos residenciales seleccionados donde se aplicó la encuesta, se realizó una observación sistemática en función de una pauta preestablecida, para registrar la presencia del Estado en los territorios, así como la presencia de fenómenos de desorden físico (basura, rayados, casas o autos abandonados, por ejemplo) y social (personas discutiendo en el espacio público, usando lenguaje vulgar, niños sin supervisión, entre otros) y una evaluación global y cualitativa de la calidad del espacio público de acuerdo a la percepción de los observadores bajo ciertos criterios de valoración estandarizados (estado de la señalética, cuidado de los jardines, sensación de temor o inseguridad al momento de hacer la observación, entre otros). Los observadores fueron estudiantes universitarios en sus últimos años de formación de pregrado, provenientes de las ciencias sociales, quienes antes de salir a terreno recibieron una capacitación ad hoc. Para los 242 barrios estudiados, se contó con cerca de 15 observadores, de tal manera que cada uno observó en promedio unos 16 barrios. Todas las observaciones fueron realizadas entre las 14 y las 17 horas entre octubre y diciembre de 2010, unas semanas luego de la aplicación de la encuesta. Para efectos de este trabajo, las variables seleccionadas de la observación sistemática dicen relación, especialmente, con la presencia del Estado en los territorios, que se registró como el número de entidades públicas de educación (preescolar y escolar), de salud (atención primaria, secundaria o terciaria), sedes sociales (juntas de vecinos, organizaciones funcionales, etcétera), equipamiento público deportivo y recreativo (canchas deportivas, parques y plazas, por ejemplo) y presencia policial circundante al sector (comisarías, subcomisarías o retenes de Carabineros de Chile y dependencias de la Policía de Investigaciones). Este conjunto de indicadores fueron puestos en relación con las consultas acerca de la satisfacción de los vecinos con los servicios que prestan dichas instituciones del Estado a nivel local.

Luego, a la base de datos de la encuesta, más los datos de las observaciones sistemáticas, se agregaron datos de localización de los conjuntos de vivienda social que fueron entregados entre 1980 y 2010 en la ciudad de Santiago. La localización de conjuntos de vivienda social correspondió a la ubicación detallada y georreferenciada de los conjuntos mencionados a partir de los archivos del Ministerio de Vivienda y Urbanismo de Chile ${ }^{5}$. Se estudió la asociación entre los fenómenos de violencia y las personas viviendo en condiciones de marginalidad, usando como proxy de esta situación, la distancia entre barrios seleccionados y conjuntos de viviendas sociales georreferenciados.

La muestra del estudio fue obtenida de la siguiente manera. Primero, se seleccionaron al azar 121 Unidades Vecinales ${ }^{6}$ (UV) sobre un universo de 885 UV en las 34 comunas urbanas de Santiago. Luego, por cada UV

\footnotetext{
5 Proyecto FONDECYT No 1100212. "Cambios espaciales en la sociogeografía del Gran Santiago: ¿Mito o Realidad?"

6 La Unidad Vecinal es una división político-administrativa del territorio de la comuna, cuyo gobierno local está en manos del Alcalde Municipal. Las Unidades Vecinales tiene por finalidad estimular la organización de los vecinos y permitir la expresión de intereses ante el municipio y otras autoridades del Estado. La Junta de Vecinos es la denominación que se da a la organización ciudadana de ese espacio subcomunal, regulada por la Ley 19.418. En Santiago de Chile existen 885 UV.
} 
seleccionada se eligieron al azar 2 conjuntos residenciales de 6 manzanas censales ${ }^{7}$ en promedio, unidad que fue creada especialmente para efectos de este estudio y que recibió la denominación de MicroBarrio (MB). Para establecer el universo de MBs, se construyó una grilla de MBs sobre las 34 comunas urbanas de la ciudad, mediante un procedimiento de iteración matemática que respetó hitos urbanos relevantes (por ejemplo, ningún MB podía incluir una avenida o calle importante que eventualmente significase la división del barrio en dos conjuntos residenciales separados de facto). Así, se conformó un universo de $8.206 \mathrm{MBs}$. En un tercer momento, por cada uno de los MBs seleccionados, se encuestaron entre 20 a 25 hogares $^{8}$, mediante un procedimiento de saltos aleatorios entre viviendas por calle. Cuando un hogar seleccionado aleatoriamente no pudo ser entrevistado, se reemplazó por otro seleccionado de manera aleatoria dentro del mismo $\mathrm{MB}$, hasta alcanzar una meta referencial de 25 hogares por MB (o eventualmente hasta agotar los hogares del $\mathrm{MB}$ ), meta que se cumplió en la gran mayoría de los casos, siendo 24,2 el promedio resultante de hogares encuestados por MB. En promedio, los MBs tienen cerca de 200 hogares y comprenden una población de 824 habitantes distribuidos en una superficie de 57.291 metros cuadrados. Es importante destacar que la muestra no tiene representatividad comunal o por zonas de la ciudad, sino que para Santiago en su conjunto, es decir, los 242 MBs y las 121 UVs encuestados representan a todos los MBs y las UVs de las comunas urbanas de Santiago.

El cuestionario se estructuró en 5 módulos, más uno que consultó las características

\footnotetext{
7 La manzana es la unidad territorial más básica con que opera el CENSO poblacional en Chile según el Instituto Nacional de Estadísticas (INE). Corresponde al espacio territorial interior comprendido en la intersección de tres o más calles, dibujando normalmente un cuadrado o rectángulo, sin que haya en este espacio una unidad menor que responda a esta misma definición.

8 El número de 25 hogares fue impuesto como una meta referencial para cada microbarrio, debido a que es un número que permite obtener estadísticas (promedios a nivel de $\mathrm{MB}$, por ejemplo) con buenas propiedades estadísticas (varianzas pequeñas, acotadas), bajo el supuesto que dichos hogares son seleccionados de manera aleatoria.
}

sociodemográficas del encuestado y del jefe de hogar. El primer módulo incluyó información básica sobre el hogar. El segundo, recogió la percepción del encuestado acerca de características relevantes del entorno residencial (elección del barrio, estabilidad residencial, satisfacción con el barrio, actividades rutinarias, trasporte público, entre otras). El tercer módulo consultó sobre presencia y efectividad del Estado (presencia y uso de instituciones de educación y salud públicas y privadas, relación con los municipios, confianza en instituciones de control formal, entre otras). El cuarto módulo recogió las percepciones del encuestado en cuanto a los recursos comunitarios que identificaba en su barrio (confianza, cohesión, frecuencia de contactos, calidad de los vínculos en el contexto barrial, participación vecinal, control social informal, identidad y adhesión a valores y normas pro sociales comunes, entre otras). El último módulo, de victimización delictual, violencia e incivilidades, consultó sobre eventos delictuales sufridos por el hogar y el encuestado -siguiendo las formas tradicionales de las encuestas de victimización-, más preguntas sobre percepción de violencia y de percepción de desorden social en el barrio.

\section{Victimización, delitos y percepción de seguridad}

Tal como se muestra en el Cuadro $N^{0} 1$, la proporción de hogares victimizados por conjuntos residenciales o MBs es en promedio de un $15 \%$, con una desviación estándar de $10 \%$. En un $5,4 \%$ de los MBs no se registró ningún hogar victimizado, mientras que la proporción de hogares victimizados más alta alcanza el $52 \%$ de hogares por MB. En relación a lo anterior, el número de delitos totales que reportan en promedio todos los hogares por $\mathrm{MB}$ es de 6,05 eventos con una desviación estándar de 5,3, siendo el número máximo 38 eventos reportados. Si se considera el lugar de ocurrencia, el $72 \%$ en promedio de delitos reportados por MB fueron localizados en los entornos residenciales y un $24 \%$ fuera de los mismos.

El número de delitos sucedidos en el barrio fue en promedio 4,46 hechos delictuales, mientras que el número de delitos ocurridos fuera del barrio tuvo por valor promedio 
Cuadro $N^{\circ} 1$

Victimización delictual y número de delitos por entornos residenciales

\begin{tabular}{|l|r|r|r|r|r|}
\hline Variable & $\begin{array}{r}\mathrm{N}^{\circ} \\
\text { obs. }\end{array}$ & Promedio & $\begin{array}{c}\text { Desviación } \\
\text { estándar }\end{array}$ & Mín. & Máx. \\
\hline $\begin{array}{l}\text { Victimización hogares (\% hogares victimizados por } \\
\mathrm{MB} \text { ) }\end{array}$ & 242 & 15 & 10 & 0 & 52 \\
\hline $\mathrm{N}^{\circ}$ delitos por MB (sumatoria de casos por MB) & 242 & 6,05 & 5,30 & 0,00 & 38,00 \\
\hline $\begin{array}{l}\text { Porcentaje delitos ocurridos en el MB sobre todos los } \\
\text { delitos reportados por MB }\end{array}$ & 229 & 72 & 28 & 0 & 100 \\
\hline $\mathrm{N}^{\circ}$ delitos en MB (sumatoria de casos por MB) & 242 & 4,46 & 4,54 & 0,00 & 34,00 \\
\hline $\mathrm{N}^{\circ}$ delitos fuera del MB (sumatoria de casos por MB) & 242 & 1,52 & 1,98 & 0,00 & 12,00 \\
\hline $\begin{array}{l}\mathrm{N}^{\circ} \text { delitos violentos en MB (sumatoria de casos por } \\
\text { MB) }\end{array}$ & 242 & 2,37 & 3,43 & 0,00 & 30,00 \\
\hline $\begin{array}{l}\mathrm{N}^{\circ} \text { delitos contra la propiedad en MB (sumatoria de } \\
\text { casos por MB) }\end{array}$ & 242 & 2,38 & 3,28 & 0,00 & 20,00 \\
\hline $\begin{array}{l}\text { Porcentaje delitos denunciados sobre delitos } \\
\text { reportados por MB }\end{array}$ & 229 & 56 & 32 & 0 & 100 \\
\hline
\end{tabular}

Fuente: Elaboración propia en función de datos Encuesta Proyecto Crimen y Violencia Urbana.

1,52. Ambas variables muestran desviaciones típicas por encima del promedio y distribuciones asimétricas positivas, lo que implica que gran cantidad de unidades residenciales presentan bajos números de delitos y que los valores promedio no deben ser tomados como representativos del conjunto. A modo de ilustración se presenta la Figura $\mathrm{N}^{0} 1$, donde es posible apreciar que hay un $10 \%$ de MBs donde no se reporta ningún delito, mientras que en cerca del $90 \%$ de los casos donde sí se registran delitos, el número de eventos oscila entre 1 y 10 experiencias de victimización por MB. Aquellos MBs donde

Figura $N^{\circ} 1$

Distribución del número de delitos ocurridos en los MB

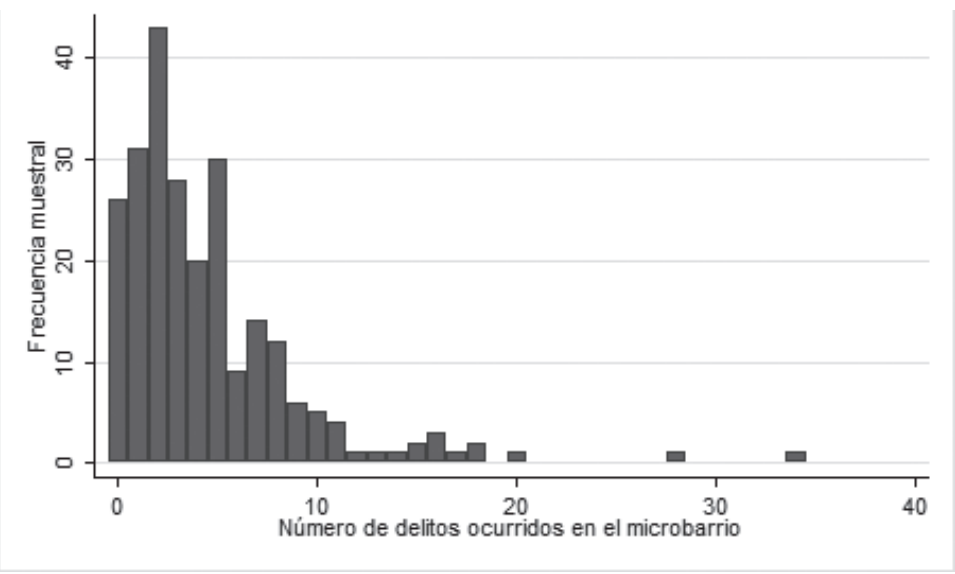

Fuente: Elaboración propia en función de datos Encuesta Proyecto Crimen y Violencia Urbana. 
el número de delitos reportados supera los 11 casos son un $5,4 \%$ de los MBs.

La victimización de hogares por delitos con la propiedad y la victimización de hogares por delitos violentos se distribuyen de manera muy similar cuando estos ocurren en el barrio. En promedio, el número de delitos violentos (tales como delitos donde ha mediado el uso de la violencia, amenaza o intimidación, por ejemplo asaltos, lesiones, homicidios, ataques sexuales u otros según el fraseo de la encuesta) por MB alcanza los 2,37 eventos, mientras que el número promedio de delitos contra la propiedad (contra la vivienda, vehículos y partes u objetos en su interior) es de 2,38, teniendo en ambos casos desviaciones típicas más altas que el promedio y valores máximos de 30 y de 20 delitos, respectivamente.
Un aspecto interesante a analizar dice relación con el porcentaje promedio de denuncia por $\mathrm{MB}$, que es de un $56 \%$ de los delitos con una desviación de $32 \%$. Este evidencia una distribución muy desigual donde un 22\% de los barrios tienen $100 \%$ de denuncia, un $10,48 \%$ de los barrios tienen un $50 \%$ de denuncia y un $9,17 \%$ de los barrios tienen $0 \%$ de denuncia, o sea, ningún delito denunciado.

Llama la atención, tal como se indica en el Cuadro $\mathrm{N}^{\circ} 2$, que en las evaluaciones de riesgo que hacen los encuestados con respecto a sus entornos residenciales, aparecen como de mayor envergadura aquellas situaciones que involucran la propiedad, ya sea la vivienda, en primer lugar, y luego, el vehículo, sus partes u objetos. Solo en tercer lugar aparece la preocupación por los asaltos en el barrio, amenazas y lesiones, respectivamente.

Cuadro $\mathrm{N}^{\circ} 2$

Percepción de riesgo delictual en el barrio, seguridad, violencia y desorden social ${ }^{9}$.

\begin{tabular}{|l|r|r|r|r|r|}
\hline Variable & $\begin{array}{r}\text { No } \\
\text { obs. }\end{array}$ & Promedio & $\begin{array}{c}\text { Desviación } \\
\text { estándar }\end{array}$ & Mín. & Máx. \\
\hline Percepción riesgo de robo vivienda en el barrio & 242 & 3,14 & 0,61 & 1,21 & 4,56 \\
\hline Percepción riesgo de robo vehículos en el barrio & 242 & 3,07 & 0,64 & 1,00 & 4,76 \\
\hline Percepción riesgo de asalto en el barrio & 242 & 3,01 & 0,61 & 1,20 & 4,74 \\
\hline Percepción riesgo de amenazas en el barrio & 242 & 2,68 & 0,69 & 1,00 & 4,52 \\
\hline Percepción riesgo de lesiones en el barrio & 242 & 2,67 & 0,66 & 1,08 & 4,68 \\
\hline Percepción de seguridad en el barrio & 242 & 3,10 & 0,46 & 1,77 & 4,31 \\
\hline Percepción de desorden social & 242 & 2,86 & 0,77 & 1,17 & 4,82 \\
\hline Percepción de violencia & 242 & 2,38 & 0,71 & 1,03 & 4,55 \\
\hline
\end{tabular}

Fuente: Elaboración propia en función de datos Encuesta Proyecto Crimen y Violencia Urbana.

\footnotetext{
9 Los datos de este cuadro, al igual que otros que contienen variables resúmenes de percepción para el conjunto de los MBs, equivalen al valor promedio de los promedios de cada MB en base a los 20 a 25 encuestados en cada uno de ellos. Las alternativas de respuesta están organizadas de 1 a 5 , donde, por lo general, el valor 5 indica una percepción más negativa o preocupante. En este cuadro la excepción es la percepción de seguridad en el barrio que fue consultada de tal modo que 5 indica mayor seguridad y 1 menor.
} 
Figura $\mathrm{N}^{\circ} 2$

Correlación entre percepción de violencia y de desorden social por MB

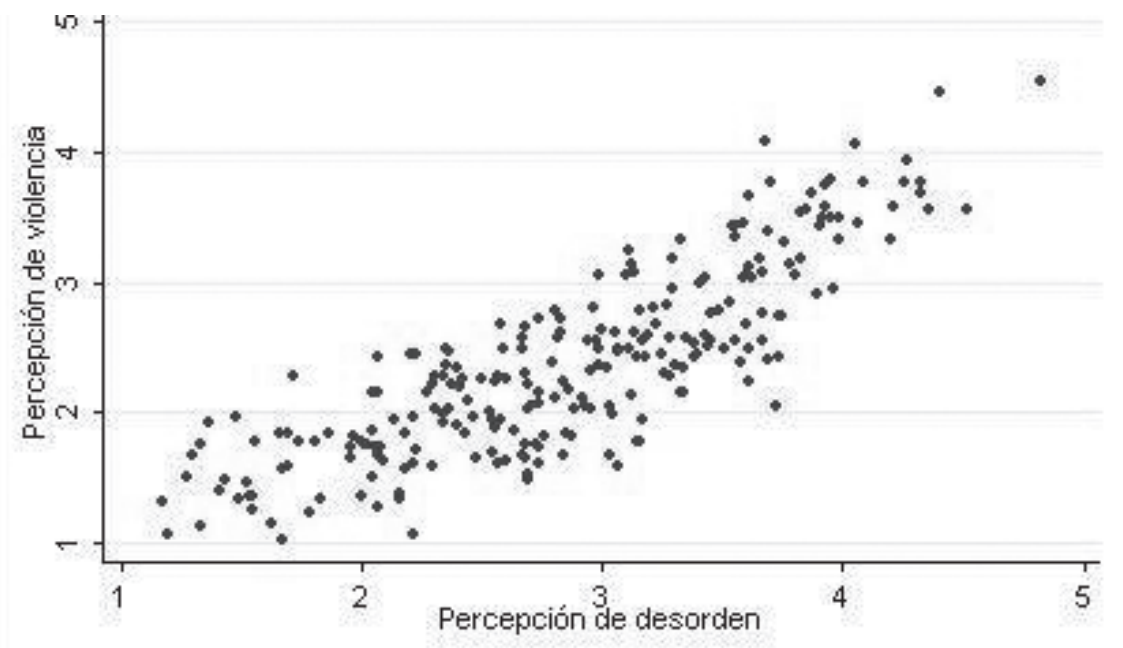

Fuente: Elaboración propia en función de datos Encuesta Proyecto Crimen y Violencia Urbana.

También se aprecia en el Cuadro $N^{\circ} 2$ que la percepción de desorden social es levemente más alta que la de violencia en el barrio, registrándose entre ambas variables una asociación notablemente alta de 0.83 , medida mediante la correlación de Spearman con un nivel de significación al 0.05, lo que se ilustra en la Figura $N^{\circ} 2$. Dado que los datos no poseen una distribución gaussiana, se usa este método libre de distribución para medir la asociación entre variables.

Por su parte, la percepción de seguridad del barrio tiene un promedio en 3,10 y desviación típica de 0,46. La percepción de desorden social muestra una media de 2,86 y desviación 0,77. La asociación entre percepción de violencia y de seguridad es significativa al 0.05 e inversa como sería esperable con un coeficiente de Spearman de -0.4409.

Tal como se muestra en el Cuadro $N^{\circ} 3$, las variables de percepción de violencia, desorden social, seguridad y las evaluaciones de riesgos delictuales en el MB están todas asociadas de manera significativa a las variables de victimización y números de delitos de diverso tipo, salvo en el caso de la asociación entre el número de delitos contra la propiedad en el barrio y la percepción de violencia. Es decir, a mayor presencia de delitos contra la propiedad ocurridos en el barrio no necesariamente aumenta la percepción de que ese barrio es más violento (medido a través de ítems tales como percepción de amenazas, riñas y enfrentamientos, asaltos con violencia, robo contra la propiedad). Las restantes relaciones entre número de delitos violentos ocurridos en el barrio, totales ocurridos en el barrio y reportados por sus vecinos, están en el sentido esperado, es decir, a mayor número de delitos -salvo los delitos contra la propiedad ya mencionados-, mayores evaluaciones de riesgo, percepción de violencia y menor percepción de seguridad. 
Cuadro $\mathrm{N}^{\circ} 3$

Coeficientes de correlación de Spearman (al 0.05) entre variables y números de delitos ${ }^{10}$

\begin{tabular}{|c|c|c|c|c|}
\hline Variable & $\begin{array}{l}N^{\circ} \text { delitos } \\
\text { reportados }\end{array}$ & \begin{tabular}{|c|}
$\mathrm{N}^{\circ}$ delitos \\
ocurridos en \\
el barrio \\
\end{tabular} & $\begin{array}{c}\mathrm{N}^{0} \text { delitos } \\
\text { violentos en } \\
\text { el barrio }\end{array}$ & $\begin{array}{c}\mathrm{N}^{0} \text { delitos } \\
\text { propiedad en } \\
\text { el barrio }\end{array}$ \\
\hline Percepción riesgo de asalto en el barrio & 0,247 & 0,267 & 0,248 & 0,218 \\
\hline $\begin{array}{l}\text { Percepción riesgo de robo vehículos en el } \\
\text { barrio }\end{array}$ & 0,200 & 0,251 & 0,151 & 0,201 \\
\hline Percepción de desorden social & 0.302 & 0.250 & 0.238 & 0.129 \\
\hline $\begin{array}{l}\text { Percepción riesgo de robo vivienda en el } \\
\text { barrio }\end{array}$ & 0,187 & 0,243 & 0,146 & 0,207 \\
\hline Percepción de violencia & 0,205 & 0,192 & 0,257 & \\
\hline Percepción riesgo de lesiones en el barrio & 0,182 & 0,178 & 0,248 & 0,143 \\
\hline Percepción riesgo de amenazas en el barrio & 0,155 & 0,128 & 0,193 & 0,139 \\
\hline Percepción de seguridad en el barrio & $-0,281$ & $-0,300$ & $-0,214$ & $-0,299$ \\
\hline Allegamiento interno (\% de hogares por MB) & 0,249 & 0,231 & 0,182 & 0,276 \\
\hline Allegamiento externo (\% de hogares por MB) & 0,199 & 0,178 & & 0,272 \\
\hline Ingresos promedio por MB (en pesos) & $-0,172$ & $-0,155$ & $-0,205$ & \\
\hline Distancia Viviendas Sociales (en metros) & $-0,184$ & $-0,133$ & $-0,155$ & $-0,212$ \\
\hline Hacinamiento (\% hogares por MB) & & & 0,178 & \\
\hline Satisfacción barrio (media MB) & $-0,228$ & $-0,209$ & $-0,216$ & \\
\hline Elección barrio (\% hogares por MB) & $-0,198$ & $-0,139$ & $-0,134$ & $-0,138$ \\
\hline Satisfacción Carabineros & $-0,1667$ & $-0,1604$ & & \\
\hline Satisfacción Municipio & $-0,1421$ & $-0,1525$ & $-0,1644$ & $-0,1857$ \\
\hline Satisfacción Ed. Básica & $-0,1421$ & & & \\
\hline Satisfacción programas municipales & $-0,1748$ & & & $-0,168$ \\
\hline Equipamiento educación & & & 0,1612 & \\
\hline Cohesión & $-0,163$ & $-0,192$ & $-0,181$ & \\
\hline Respeto a normas & & & $-0,132$ & \\
\hline Control social informal & & & $-0,130$ & \\
\hline Interacción recíproca & $-0,134$ & $-0,186$ & & $-0,137$ \\
\hline
\end{tabular}

Fuente: Elaboración propia en función de datos Encuesta Proyecto Crimen y Violencia Urbana.

\footnotetext{
10 En este cuadro solo se han incluido las variables cuyos coeficientes de correlación de Spearman arrojan significativos a 0.05 . Se han ordenado por grupo de variables y luego en función de la fuerza de la relación con respecto al número de delitos ocurridos en el barrio ( $3^{\mathrm{a}}$ columna).
} 


\section{Concentración de desventajas económicas y variables habitacionales}

Los conjuntos de viviendas sociales construidos en Santiago de Chile a partir de la década de los años ochenta hasta hoy, aglutinan población pobre, donde es razonable suponer que se desarrollan problemas delictuales asociados con la exclusión social y segregación residencial. Por ello, es que en este trabajo se incluyó esta variable de proximidad entre los MBs y los conjuntos de viviendas sociales, de modo de evaluar si es que esta está asociada con perfiles particulares en los resultados de la encuesta. La Figura $N^{\circ} 3$ muestra la localización de los conjuntos de viviendas sociales construidos entre 1980 y 2010. Es posible apreciar que estos se encuentran más bien en la periferia de la ciudad hacia el sur y el norponiente.

Figura $\mathrm{N}^{\circ} 3$

Localización de conjuntos de vivienda social en Santiago de Chile. Período 1980-2010 (en negro los conjuntos de viviendas sociales)

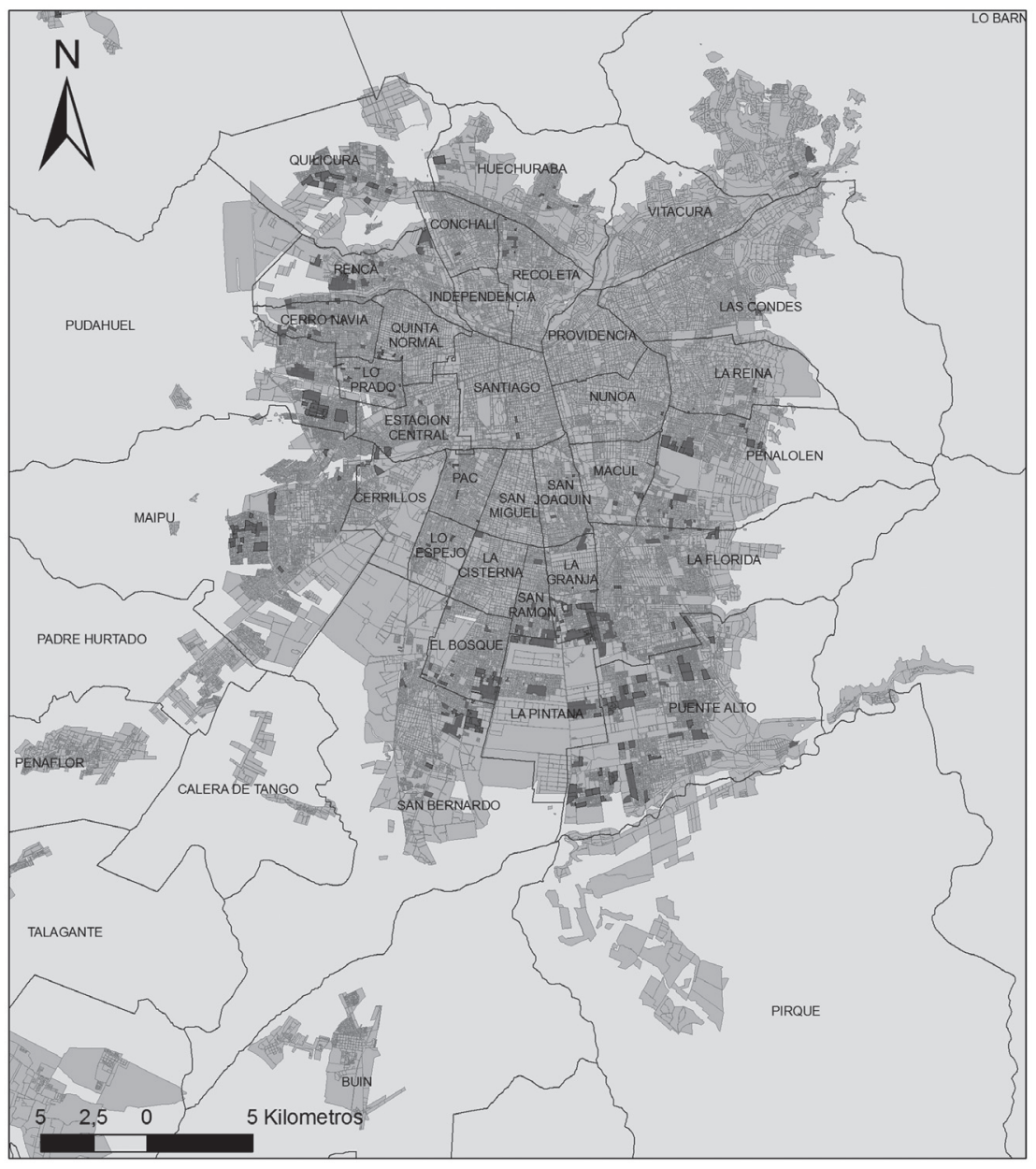

Fuente: Elaboración propia a partir de antecedentes del Ministerio de Vivienda y Urbanismo. Chile. 
Cuadro $\mathrm{N}^{\circ} 4$

Variables de concentración de desventajas económicas-habitacionales y variables residenciales

\begin{tabular}{|l|r|r|r|r|r|}
\hline Variable & $\begin{array}{c}\text { N } \\
\text { obs. }\end{array}$ & Promedio & $\begin{array}{c}\text { Desviación } \\
\text { estándar }\end{array}$ & Mín. & Máx. \\
\hline Allegamiento interno (\% de hogares por MB) & 242 & 10 & 13 & 0 & 64 \\
\hline Allegamiento externo (\% de hogares por MB) & 242 & 8 & 13 & 0 & 72 \\
\hline Ingresos promedio por MB (en pesos) & 238 & 353.911 & 253.593 & 90.000 & 2.100 .500 \\
\hline Distancia viviendas sociales (en metros) & 242 & $1.573,67$ & $1.088,27$ & 38,00 & $5.300,00$ \\
\hline Hacinamiento (\% hogares por MB) & 242 & 6 & 6 & 0 & 50 \\
\hline Satisfacción barrio (media MB) & 242 & 4,10 & 0,54 & 1,60 & 5,00 \\
\hline Elección barrio (\% hogares por MB) & 242 & 81 & 19 & 16 & 100 \\
\hline Años residencia promedio por MB (en años) & 242 & 22,16 & 7,21 & 0,68 & 47,04 \\
\hline
\end{tabular}

Fuente: Elaboración propia en función de datos Encuesta Proyecto Crimen y Violencia Urbana.

En términos descriptivos, el Cuadro $\mathrm{N}^{\circ} 4$ resume los datos para las variables que a continuación son analizadas en función con las asociaciones con los números de delitos por MB según tipo, provenientes del Cuadro $\mathrm{N}^{\circ} 3$.

El nivel de allegamiento interno, medido como el porcentaje de hogares por MB donde se evidencian más de un hogar compartiendo una misma vivienda, se asocia positivamente con el número de delitos reportados por el barrio, con el número de delitos sucedidos en el barrio, violentos y contra la propiedad. El allegamiento externo, medido como más de una vivienda en un mismo sitio, se asocia también positivamente a todas las anteriores, salvo el número de delitos violentos acontecidos en el barrio. Contrariamente, el nivel de hacinamiento, medido como el porcentaje de hogares por $\mathrm{MB}$ donde se registran 3 o más personas por pieza destinada a dormitorio en la vivienda, solo está relacionado de manera positiva con el número de delitos violentos sucedidos en el barrio. El sentido de estas asociaciones es positiva, como es esperable.

El nivel de ingresos promedio y la proximidad con viviendas sociales se asocian al número de delitos reportados por el barrio, ocurridos en el mismo barrio y al número de delitos violentos sucedidos en el barrio. Las relaciones en ambos casos son de signo inverso, es decir, a mayor ingreso, menor número de delitos reportados por el barrio y que se efectuaron en el barrio, especialmente en el caso de los delitos violentos acontecidos en el barrio. $Y$ a mayor proximidad con viviendas sociales, también el número de delitos reportados por y ocurridos en el barrio, aumentan, en una asociación leventemente superior cuando se trata de los delitos contra la propiedad. Es importante destacar que el número de delitos contra la propiedad acaecidos en el barrio solo se asocia a mayor proximidad con las viviendas sociales y no con el nivel de ingreso. Por ende, es posible hipotetizar que en sectores de menos ingresos económicos y más próximos a los conjuntos de viviendas sociales, se registran más delitos en general, siendo llamativo que el nivel de ingreso no se asocie con el número de delitos contra la propiedad, mientras que sí la proximidad a las viviendas sociales.

Con respecto a las variables de hacinamiento interno y externo, allegamiento, proximidad con las viviendas sociales y nivel de ingresos del $M B$, es relevante mencionar que están todas asociadas entre sí de manera significativa. Las asociaciones son en los sentidos previsibles: por ejemplo, a menores ingresos se asocia más cercanía con las viviendas sociales, mientras que a menores ingresos más hacinamiento o allegamiento interno o externo. Lo mismo ocurre con la proximidad a las viviendas sociales. 
Años de residencia no está asociada con ninguno de los tipos de delitos por lugar de ocurrencia. El porcentaje de hogares que ha elegido su lugar de residencia sí está asociado con todas las variables de tipos de delitos según lugar del suceso. Es decir, a mayor números de delitos reportados y/o ocurridos en el barrio se verifica una asociación negativa, en términos de que a menor elección del barrio, mayores delitos.

En cuanto al nivel de satisfacción residencial también se da una asociación negativa con el número de delitos reportados por el barrio, ocurridos en el mismo, incluido los delitos violentos, pero no con aquellos contra la propiedad acontecidos en el barrio. Es decir, la presencia de delitos contra la propiedad en el barrio no se relaciona con la satisfacción residencial, mientras que sí lo hacen los delitos violentos efectuados en el barrio.

Resulta interesante que los años de residencia no están asociados a la satisfacción residencial, pero a más años en promedio de residencia en el barrio, se asocia un menor porcentaje de hogares que eligieron el lugar de su vivienda, esto es, a más años en promedio por $\mathrm{MB}$ de residencia en el mismo barrio, menor proporción de hogares que han elegido vivir allí. También, como podría ser esperable, hay una asociación positiva entre elección del barrio y satisfacción residencial. Con respecto a las relacionadas con la percepción de seguridad, violencia y desorden social así como con las evaluaciones de riesgo delictual en el barrio, los años de residen- cia nuevamente no tienen ninguna asociación significativa.

La elección del barrio y la satisfacción residencial muestran una relación inversa con percepción de violencia y de desorden social y una relación positiva con percepción de seguridad, como sería esperable. Las evaluaciones de riesgos delictuales consultadas se relacionan negativamente con la satisfacción residencial. Finalmente, la evaluación de riesgos delictuales en general y la de lesiones se relacionan negativamente con la elección del barrio.

\section{Presencia y satisfacción con los servicios estatales}

Con respecto a las variables de presencia de las instituciones del Estado en el territorio y la satisfacción de los usuarios de sus servicios, los resultados descriptivos se muestran en el Cuadro $\mathrm{N}^{0}$ 5. Luego, tal como se evidencia en el Cuadro $N^{\circ} 3$, excluyendo la variable satisfacción con la con la Policía de Investigaciones (PDI) de las correlaciones por presentar un número de observaciones de solo 37, Ilama la atención que la satisfacción con los servicios municipales está inversamente relacionada con el número de delitos, reportados, ocurridos en el barrio y ya sean violentos o contra la propiedad. Además, una menor satisfacción con los programas municipales también está relacionada con un mayor número de delitos reportados y un mayor número de delito contra la propiedad acaecidos en el barrio.

Cuadro $\mathrm{N}^{\circ} 5$

Variables de presencia de instituciones estatales en los MB y de satisfaccion con sus servicios ${ }^{11}$.

\begin{tabular}{|l|r|r|r|r|r|}
\hline Variable & $\begin{array}{c}\mathrm{N}^{\circ} \\
\text { obs. }\end{array}$ & Promedio & $\begin{array}{c}\text { Desviación } \\
\text { estándar }\end{array}$ & Mín. & Máx. \\
\hline Satisfacción Carabineros & 232 & 3,29 & 0,30 & 2,33 & 4,33 \\
\hline Satisfacción Ed. Básica & 242 & 3,47 & 0,38 & 2,31 & 4,75 \\
\hline Satisfacción Ed. Media & 242 & 3,42 & 0,45 & 1,36 & 4,67 \\
\hline
\end{tabular}

\footnotetext{
${ }^{11} \mathrm{Al}$ igual como ya fuera señalado, los datos de las variables de satisfacción con la atención de los servicios públicos se obtuvieron en base a los promedios por MB de los 20 a 25 encuestados en cada uno de ellos, de acuerdo a preguntas organizadas de 1 a 5 , donde en estos casos el valor 5 indica la mejor evaluación posible
} 
Continuación Cuadro No 5

\begin{tabular}{|l|r|r|r|r|r|}
\hline Variable & $\begin{array}{r}\text { No } \\
\text { obs. }\end{array}$ & Promedio & $\begin{array}{c}\text { Desviación } \\
\text { estándar }\end{array}$ & Mín. & Máx. \\
\hline Satisfacción Municipio & 229 & 3,30 & 0,56 & 1,00 & 4,67 \\
\hline Satisfacción programas municipales & 237 & 3,66 & 0,49 & 2,17 & 5,00 \\
\hline Satisfacción PDI & 37 & 3,29 & 0,30 & 2,33 & 4,00 \\
\hline Satisfacción Salud Primaria & 242 & 3,19 & 0,48 & 1,91 & 4,63 \\
\hline Satisfacción Salud Secundaria & 238 & 3,17 & 0,53 & 2,00 & 4,59 \\
\hline Equipamiento de educación (número por MB) & 241 & 0,62 & 0,80 & 0,00 & 4,00 \\
\hline Equipamiento policial (número por MB) & 241 & 0,08 & 0,28 & 0,00 & 1,00 \\
\hline Equipamiento recreación deportivo (número por MB) & 241 & 1,23 & 1,41 & 0,00 & 8,00 \\
\hline Equipamiento de salud (número por MB) & 241 & 0,16 & 0,39 & 0,00 & 2,00 \\
\hline Equipamiento social (número por MB) & 241 & 0,41 & 0,52 & 0,00 & 2,00 \\
\hline
\end{tabular}

Fuente: Elaboración propia en función de datos Encuesta Proyecto Crimen y Violencia Urbana.

A su vez, la satisfacción en la atención de Carabineros de Chile está relacionada inversamente con el número de delitos reportados y ocurridos en el barrio, sin influir se trate de delitos violentos o contra la propiedad (ver Cuadro $\mathrm{N}^{\circ} 3$ ). El porcentaje de delitos denunciados por $\mathrm{MB}$ no está asociado a ninguna de las variables de presencia y satisfacción con las instituciones estatales, ni con las relacionadas con los niveles de percepción de in/seguridad en el barrio, ni con las denominadas de concentración de desventajas económicashabitacional.

\section{Variables sociales a nivel del barrio}

Las variables sociales muestran una homogeneidad entre MBs, con medias centradas, rangos pequeños y reducida variabilidad, tal como muestra el Cuadro $N^{\circ} 6$.

\section{Cuadro $\mathrm{N}^{\circ} 6$}

Variables sociales a nivel de MB

\begin{tabular}{|l|r|r|r|r|r|}
\hline Variable & $\begin{array}{c}\text { N. } \\
\text { obs. }\end{array}$ & Promedio & $\begin{array}{c}\text { Desviación } \\
\text { estándar }\end{array}$ & Mín. & Máx. \\
\hline Cohesión & 242 & 3,49 & 0,30 & 2,52 & 4,46 \\
\hline Control social informal & 242 & 2,83 & 0,68 & 1,30 & 4,38 \\
\hline Percepción de integración/exclusión & 242 & 5,52 & 1,15 & 2,24 & 8,76 \\
\hline Interacción recíproca & 242 & 3,16 & 0,52 & 1,46 & 4,58 \\
\hline Respeto a normas & 242 & 4,17 & 0,33 & 3,24 & 5,00 \\
\hline Organización local para seguridad (\% promedio por MB) & 242 & 17 & 18 & 0,00 & 84 \\
\hline Voluntad supervisión niños (\% promedio por MB) & 242 & 28 & 22 & 0,00 & 84 \\
\hline
\end{tabular}

Fuente: Elaboración propia en función de datos Encuesta Proyecto Crimen y Violencia Urbana. 
Como ya mostró el Cuadro $\mathrm{N}^{\circ} 3$, el nivel de cohesión social entre vecinos en el entorno residencial se asocia negativamente con el número de delitos reportados, con el número de delitos sucedidos en el barrio $y$, específicamente, con el número de delitos violentos efectuados en el barrio. Mientras, el control social informal y el respeto a normas solamente están relacionados con el número de delitos violentos ocurridos en el barrio. Al contrario, los intercambios recíprocos entre vecinos se relacionan inversamente con el número de delitos reportados y acontecidos en el barrio, específicamente, contra la propiedad, pero no con los delitos violentos acaecidos en el barrio. Es decir, el control social informal y el respeto a normas se encuentran disminuidos precisamente donde hay más delitos violentos, lo que es coherente con los antecedentes presentados por la Teoría Ecológica del Delito y los estudios de Sampson. En este sentido, los lazos sociales más superficiales (intercambios recíprocos) serían útiles para controlar los delitos contra la propiedad pero no los delitos violentos sucedidos en el barrio.

Con respecto a las relaciones entre las variables sociales del barrio y las de percepción de seguridad en el territorio y otras afines, la cohesión está relacionada con todas, salvo con la percepción de seguridad, lo que es interesante, ya que se esperaría que la cohesión mejorara o se relacionara positivamente con la percepción de seguridad. En este caso, a mayor cohesión, menor evaluación de riesgo y de percepción de violencia y de desorden social, pero no necesariamente se da mayor percepción de seguridad. Esta podría ser algo que va más allá de la mera percepción de violencia y desorden social. Por su parte, el control social informal no presenta ninguna asociación significativa con estas últimas variables de percepción.

Los intercambios recíprocos y el respeto de normas presentan algunas asociaciones significativas con las variables relacionadas con la inseguridad. Los intercambios recíprocos se relacionan inversamente con la evaluación de riesgo de asaltos y riesgo de delitos contra la propiedad (vehículos y vivienda). Mientras que el respeto a normas se relaciona también inversamente con riesgo general de amenazas delictuales, de lesiones y con percepción de violencia y de desorden social. Por su parte, la voluntad de supervisar niños y jóvenes se relaciona inversamente con la percepción de riesgo general y en específico con la evaluación de riesgo ante delitos contra la propiedad (vehículos y vivienda), pero no tiene asociación con percepción de desorden social o violencia. Y finalmente, la percepción de exclusión se relaciona inversamente con riesgo de asalto, percepción de desorden y de violencia.

En suma, entre las variables relativas a las relaciones sociales entre vecinos y los indicadores de números de delitos, hay algunas asociaciones significativas. Al respecto, es notable que ciertas variables sociales se asocian a ciertos tipos de delitos y no a otros (ver Cuadro $\mathrm{N}^{\circ} 3$ ). Por ejemplo, el nivel de cohesión entre vecinos, el respeto a normas y el control social informal se asocian a delitos violentos ocurridos en el barrio, pero no a delitos contra la propiedad ocurridos en el mismo. Por su parte, el nivel de interacción recíproca (intercambio de favores) entre vecinos está asociado a los delitos contra la propiedad en el barrio, pero no con los delitos violentos ocurridos en este. Ello resulta interesante ya que ambas variables, cohesión e interacción recíproca, aparecen asociadas significativamente al número de delitos reportados por y ocurridos en el barrio, teniendo cada una asociación solo con un tipo de delitos efectuados en el barrio, lo que podría estar dando cuenta de cualidades diferenciales en las relaciones sociales de los vecinos en la medida de que en sus territorios hay más delitos de un tipo que de otro.

Con respecto a las variables de concentración de desventajas económicas y habitacionales y aquellas relativas a las relaciones sociales entre vecinos, se observa una asociación positiva entre ingresos económicos y control social informal, además de con una mejor disposición a organizarse en pro de la seguridad del barrio y a supervisar a niños y jóvenes, aunque estos no sean familiares. Del mismo modo, aparece una asociación positiva entre ingresos y percepción de inclusión social. Ahora bien, los barrios con menos ingresos y más próximos a viviendas sociales son percibidos por sus vecinos con mayores niveles de desorden social.

El hacinamiento al interior de la vivienda aparece asociado negativamente a la cohesión 
social y al respeto a normas, así como con la percepción de inclusión social, registrando también una asociación positiva con mayores niveles de desorden social en el barrio.

\section{Discusión y conclusiones}

Tomando como unidad de análisis los $M B$ o entornos residenciales de la ciudad de Santiago incluidos en esta investigación, en promedio, en un $15 \%$ de hogares que componen un barrio hay algún miembro que fue víctima de un delito entre agosto de 2009 y octubre de 2010. Casi el $72 \%$ de esas experiencias de victimización ocurrieron en las inmediaciones de su barrio, lo que resulta llamativo a efectos de sugerir cursos de acción preventivos. Los lugares de ocurrencia de los delitos sería para las víctimas relativamente habituales en sus rutinas $y$, por ende, conocidos, frente a lo cual no sería extraño que se instalaran comportamientos de evitación de los mismos. La proporción de delitos denunciados por MBs es un promedio de un $56 \%$, indicador asociado positivamente al porcentaje de elección del barrio, al nivel de intercambios recíprocos entre vecinos en el barrio y a la percepción de inclusión social. Se constata que la denuncia no pasa entonces por el estatus económico de las víctimas, sino que la denuncia de delitos a nivel de barrio se relaciona con otras dimensiones del mismo de carácter más social o simbólico, como es la percepción de inclusión social.
Estos datos son relativamente consistentes con los que arroja la Encuesta Nacional de Seguridad Urbana (ENUSC) del año 2010, realizada por el Ministerio del Interior y el Instituto Nacional de Estadísticas, para las comunas urbanas de la Región Metropolitana (cuya superficie es prácticamente equivalente a la superficie considerada en este estudio). Ahora bien, la ENUSC tiene por unidad de observación los hogares, y no los barrios o entornos residenciales, como es el caso del presente estudio, y tiene representatividad comunal para ciertas comunas de especial interés para las políticas públicas que se elaboran a nivel central, especialmente las de más bajos recursos. Habiendo hecho esta salvedad, el porcentaje de hogares victimizados según la ENUSC entre septiembre 2009 y diciembre 2010 fue de 29,5\% para la Región Metropolitana, indicador que no resulta directamente comparable con el promedio de un $15 \%$ de los hogares de un barrio que fueron victimizados en igual período según este estudio. El porcentaje de denuncia entre ambas fuentes no resulta relativamente similar; mientras la ENUSC 2010 arroja un 34\%, el presente estudio arrojó un $56 \%$. Con respecto al lugar de ocurrencia de los delitos, el presente estudio arrojó que aproximadamente un $72 \%$ de los delitos ocurrieron en las inmediaciones del barrio, lo que puede ser complementado con la ENUSC que consulta el lugar de ocurrencia del delito según su tipo y procedencia o estrato socioeconómico de la víctima, tal como se muestra en el Cuadro $N^{\circ} 7$.

Cuadro $\mathrm{N}^{\circ} 7$

Porcentaje relativo de delitos ocurridos en el barrio según tipo de delitos y estrato socioeconómico ${ }^{12}$

\begin{tabular}{|l|r|r|r|r|r|r|}
\hline & ABC1 & C2 & \multicolumn{1}{c|}{ C3 } & D & E & RM \\
\hline Robos o Hurtos de Vehículo & 52,9 & 54,1 & 55,8 & 26,2 & 100,0 & 47,9 \\
\hline Robos o Hurtos desde Vehículo & 37,0 & 38,7 & 54,9 & 62,6 & 9,9 & 51,3 \\
\hline Robo con Violencia o Intimidación en las Personas & 0,0 & 3,7 & 24,5 & 36,5 & 36,4 & 28,9 \\
\hline Hurto & 12,9 & 21,6 & 15,4 & 28,8 & 68,9 & 22,8 \\
\hline Lesiones & 0,0 & 17,5 & 23,8 & 52,2 & 49,8 & 39,6 \\
\hline
\end{tabular}

Fuente: ENUSC, $2010^{13}$.

\footnotetext{
$12 \mathrm{El}$ instrumento de la ENUSC consulta por cada evento de victimización el lugar de ocurrencia, para lo cual da como alternativas a) "En o frente de la propia casa o en su barrio"; b) En otra parte de la comuna donde vive; c) En otra comuna de la región donde vive (cuál); d) En otra región del país; e) En el extranjero y No Sabe y No Responde. En el Cuadro $\mathrm{N}^{\circ} 7$ se han tomado solamente los porcentajes relativos de la primera alternativa.

13 Disponible en http://www.dsp.gov.cl/enusc_2010.html, consultado el 4 de octubre 2013.
} 
Del Cuadro $N^{\circ} 7$ llama la atención que en general el delito más frecuente en las inmediaciones de la residencia sea el robo o hurto de un objeto desde el vehículo (con un $51,3 \%$ ), seguido del robo o hurto del vehículo completo (con un $47,9 \%$ ), el que tuvo lugar en un $100 \%$ en el barrio para el estrato socioeconómico más bajo. Del mismo modo, para el estrato más bajo, el 68,9\% de los hurtos, seguido del $49,8 \%$ de las lesiones y del $36,4 \%$ de los robos con violencia e intimidación han sucedido en el barrio. Para el estrato más rico, Ilama la atención la ausencia total reportada de lesiones y robos con violencia e intimidación en el barrio. No obstante, el barrio como lugar de victimización violenta (lesiones más robo con violencia) va aumentando en la medida en que decrece el nivel socioeconómico. Estos datos ENUSC 2010 son congruentes con una mayor percepción de violencia en los barrios más pobres en este estudio.

En este sentido, la percepción de violencia en un barrio aparece relacionada con el delito violento pero no con el delito contra la propiedad, como ya se mencionó en el análisis de los Resultados. Es decir, un barrio con un alto número de delitos contra la propiedad no necesariamente es percibido como más violento. Es posible hipotetizar que habría una distinción entre los vecinos consultados en términos de barrios violentos $y$, posiblemente, barrios inseguros, en el sentido de que los delitos contra la propiedad no inciden en el nivel de violencia con que se considera al barrio. Es importante subrayar que la cohesión social (lazos fuertes) pareciera no tener efectos sobre la percepción de seguridad, a pesar de que a más cohesión, se asocian menos delitos violentos ocurridos en el barrio. Por su parte, un mayor nivel de intercambios recíprocos (lazos débiles), aparece asociado a menos delitos contra la propiedad.

Llaman la atención algunos indicadores que refleja el estudio en materia de estabilidad residencial (en promedio, los hogares ocupan la misma vivienda hace 22 años), la satisfacción residencial (en promedio es de 4,1 en una escala que va de 1 a 5) y el porcentaje de hogares por barrio que en promedio dicen haber elegido su barrio, el que alcanza a un $81 \%$. Ahora bien, los años de residencia promedio y la satisfacción resi- dencial promedio en los barrios no arrojaron asociación alguna, no obstante, a más años de residencia, menor proporción de hogares que han elegido su lugar de residencia. Los estratos más bajos acuden al sistema de viviendas sociales para adquirir una vivienda y en la mayoría de los casos tienen pocas posibilidades de elección del lugar de residencia y de mudarse a otra vivienda, salvo en algunas excepciones. Por ello, no resulta incomprensible que donde haya menos satisfacción residencial, también los hogares hayan tenido menos opciones de elección y lleven más años ocupando la misma vivienda.

Se confirma una vez más en este estudio cómo los conjuntos de viviendas sociales y la proximidad a los mismos tienen relación con el delito, especialmente, con el delito contra la propiedad. Y tal como sugieren Rodríguez y Sugranyes (2005), también con el delito violento toda vez que, además, el hacinamiento se relaciona específicamente con el delito violento y no con el delito contra la propiedad. Adicionalmente, el nivel de ingresos está efectivamente asociado con el número de delitos reportados por y sucedidos en el barrio, específicamente con los delitos violentos. En otras palabras, en este estudio se confirma la asociación entre concentración de desventajas socioeconómicas y habitacionales, y los niveles de los delitos que implican violencia interpersonal.

Estos resultados evidencian que un enfoque ecológico del delito, que considere dinámicas que exceden la simple relación entre delincuente y víctima, produce evidencia útil en Santiago de Chile. Sugerencias para una nueva política pública requieren un estudio de estos datos con un modelamiento capaz de testear hipótesis, lo cual será materia de un segundo trabajo.

\section{Limitaciones del estudio}

Este estudio no considera más que una medición, por lo que no alcanza a captar variaciones temporales. Y una de las dimensiones cruciales en el análisis de violencia es su dimensión histórica que dé cuenta de la evolución de conglomerados. En el caso de la tipificación de los $\mathrm{MB}$, en el presente estudio, realizados mediante una técnica de georreferenciación, la ausencia de un estudio 
histórico que entienda cómo esas entidades efectivamente se han aglutinado, representa una limitación del estudio.

Además, el trabajo es de carácter descriptivo y opera con datos muestrales. Dada la forma en que se obtuvo la muestra, de manera de utilizar como unidad de análisis al barrio o entorno residencial, es importante tener cierto cuidado en la interpretación de los datos. Además, la inconsistencia de los factores de expansión para MBs no permite extrapolar estos resultados a la población del universo. Sin embargo, como ya fuera enunciado, es necesario avanzar en la investigación interdisciplinaria sobre delincuencia y violencia, utilizando unidades de análisis territorial más reducidas, para las cuales no existen datos disponibles a nivel exhaustivo. En este sentido, es posible que estos hallazgos sean una fuente de inspiración para futuras investigaciones empíricas y para contribuir a una línea de investigación "barrializada" acerca de estos fenómenos.

\section{Referencias bibliográficas}

ARAYA, J. y SIERRA, D., Índice de vulnerabilidad social - delictual. Santiago de Chile: Ministerio del Interior, 2002.

ARIAS, D. Drugs and democracy in Rio de Janeiro: trafficking, social networks, and public security. Durham: Duke University Press, 2006.

ARRIAGADA, I. y GODOY, L. ¿Prevención o represión? El falso dilema de la seguridad ciudadana. Revista de la CEPAL, 2000, N 70 , p. 110-136.

BURSIK, R. Social disorganization and theories of crime and delinquency: problems and prospects. Criminology, 1988, Vol. 26, № 4, p. 519-552.

BUVINIĆ, M.; MORRISON, A. y SHIFTER, M. La Violencia en América Latina y el Caribe: Un Marco de Referencia para la Acción Banco Interamericano de Desarrollo. Washington, D.C.: Banco Interamericano de Desarrollo, 1999.

CARRIÓN, F. De la violencia urbana a la convivencia ciudadana. En: BOBEA, L. (editor). Entre el crimen y el castigo. Seguridad ciudadana y control democrático en América
Latina y el Caribe. Caracas: Nueva Sociedad, 2003, p. 51-84.

COHEN, L. \& FELSON, M. Social Change and Crime Rate Trends: A Routine Activity Approach. American Sociological Review, 1979, $N^{\circ} 44$, p. 588-605.

CONCHA-EASTMAN, A. y GUERRERO, R. Vigilancia epidemiológica para la prevención y el control de la violencia en las ciudades. Revista Panamericana de Salud Pública, 1999, Vol. 5, p. 322-331.

ESCOBAR, G. El uso de la teoría de la desorganización social para comprender la distribución de homicidios en Bogotá, Colombia. Revista INVI, 2012, Vol. 27, N 74, p. 21-86.

FAJNZYLBER, P.; LEDERMAN, D. \& LOAYZA, N. Determinants of crime rates in Latin America and the world: An empirical assessment. Washington, D.C.: The World Bank, 1998.

GAVIRIA, A. \& PAGÉS, C. Patterns of crime victimization in Latin America. Journal of Development Economics, 2002, Vol. 67, No 1, p. 181-203.

KAZTMAN, R.; ÁVILA, S.; BARAIBAR, X.; CORBO, G.; FILGUEIRA, F.; ERRANDONEA, F.; FURTADO, M.; GELBER, D.; RETAMOSO, A. y RODRÍGUEZ, F. La Ciudad fragmentada: Respuesta de los sectores populares urbanos a las transformaciones del mercado y del territorio en Montevideo. Montevideo: Instituto de Investigación sobre Integración, Pobreza y Exclusión Social, IPES, 2003.

LONDOÑO, J. Pobreza, Desigualdad y Formación del Capital Humano 1950-2025: Estudios del Banco Mundial sobre América Latina y el Caribe. Washington, D.C.: Banco Mundial, 1996.

MANZANO, L. Violencia en Barrios Críticos. Explicaciones teóricas y estrategias de intervención basadas en el papel de la comunidad. Santiago de Chile: RIL Editores CESC, 2009.

MAZEROLLE, L.; WICKES, R. \& MC BROOM, J. Community Variations in Vio- 
lence: the role of social ties and collective efficacy in comparative context. Journal of Research in Crime and Delinquency, 2010, $\mathrm{N}^{\circ} 47$, p. 3-30.

MONCADA, E. The Politics of Urban Violence: Challenges for Development in the Global South. Studies in Comparative International Development, 2013, Vol. 48, № 3, p. 217-239.

MOSER, C. \& MCILWAINE, C. Encounters with violence in Latin America: urban poor perceptions from Colombia and Guatemala. London: Routledge; 2004.

NÚÑEZ, J.; TOCORNAL, X. y HENRÍQUEZ, P. Determinantes individuales y del entorno residencial en la percepción de seguridad en barrios del Gran Santiago, Chile. Revista INVI, 2012, Vol. 27, Nº 74, p. 87-120.

OLAVARRÍA, M.; TOCORNAL, X.; MANZANO, L. y FRUHLING, H. Crimen y violencia urbana. Aportes de la ecología del delito al diseño de políticas. Revista INVI, 2008, Vol. 23, No 64, p. 19-59.

PORTES, A. y HOFFMAN, K. Las estructuras de clase en América Latina: composición y cambios durante la época neoliberal. Santiago de Chile: CEPAL, 2003.

ROCHÉ, S. Tolérance Zéro? Incivilités et Insécurité. Paris: Edition Odile Jacob, 2004.
RODRÍGUEZ, A. y SUGRANYES, A. Vivienda social y violencia intrafamiliar: una relación inquietante. Revista INVI, 2005, Vol. 20, No 53, p. 11-19.

SAMPSON, R. Great American City. Chicago and the enduring neighborhood effect. Chicago: The University of Chicago Press, 2013.

SAMPSON, R. The Community. In: WILSON, J.Q. \& PETERSILI, J. Crime. San Francisco: Institute for the Contemporary Studies, 1995.

SAMPSON, R.; MORENOFF, J. \& GANNON-ROWLEY, T. Assessing Neighborhood Effects: Social Processes and New Directions in Research. Annual Review of Sociology, 2002, N²8, p. 443-478.

SHAW, C. \& MCKAY, H. Juvenil Delinquency and Urban Areas. Chicago: University of Chicago Press, 1942.

VILLARREAL, A. \& DA SILVA, B. Social Cohesion, Criminal Victimization and Perceived Risk of Crime in Brazilian Neighborhoods. Social Forces, 2006, Vol. 84, Nº 3, p. 1725-1753.

WORLD BANK. Violence in the City Understanding and Supporting Community Responses to Urban Violence. Washington, D.C.: The World Bank, 2010. 
